\title{
ON ONE-PARAMETER SEMI-GROUPS OF LINEAR TRANSFORMATIONS
}

\author{
R. S. PHILLIPS
}

Let $X$ be a Banach space and let $\mathbb{E}(X)$ be the Banach algebra of bounded linear transformations on $X$ to itself. A one-parameter semigroup of operators in $\mathbb{E}(X)$ is defined to be a function $T(\xi)$ on $(0, \infty)$ to $\mathbb{E}(X)$ such that

$$
T\left(\xi_{1}+\xi_{2}\right) x=T\left(\xi_{1}\right) T\left(\xi_{2}\right) x
$$

for $0<\xi_{1}, \xi_{2}<\infty$ and $x \in X . T(\xi)$ is said to be weakly measurable if $f[T(\xi) x]$ is a measurable numerically-valued function for each $x \in X$ and $f \in \bar{X} . T(\xi)$ is said to be strongly measurable if, for each $x, T(\xi) x$ is the limit almost everywhere of a sequence of step functions (see [2, pp. 36-38] ${ }^{1}$ ).

Dunford [1] and later Hille [2, pp. 183-184] showed that if $T(\xi)$ is strongly measurable and if $\|T(\xi)\|$ is bounded in each interval $[\delta, 1 / \delta]$, then $T(\xi) x$ is continuous for $\xi>0$. We shall show that the first hypothesis for this theorem implies the second. We shall also show by means of an example that weak measurability is not sufficient to imply the boundedness of $\|T(\xi)\|$ in any interval $[\delta, 1 / \delta]$.

We being by proving the following lemma.

LeMMA 1. Let $T(\xi)$ be a strongly measurable one-parameter semi-group of operators in $\mathbb{E}(X)$ and let $x_{0} \in X$, then there exists a separable closed linear subspace $X_{0}$ of $X$ containing $x_{0}$ and a set $E_{0}$ of measure zero such that $T(\xi) x \in X_{0}$ for every $x \in X_{0}$ if only $\xi \notin E_{0}$.

As $T(\xi) x_{0}$ is strongly measurable, there will exist a set $F_{0}$ of measure zero such that $\left[T(\xi) x_{0} \mid \xi \notin F_{0}\right]$ is separable valued. We define

$$
X_{0} \equiv \text { linear closed extension of }\left[x_{0}, T(\xi) x_{0} \mid \xi \notin F_{0}\right] .
$$

Then $X_{0}$ is a separable closed linear subspace of $X$. Further there will be a denumerable set

$$
S=\left[x_{0}, T\left(\xi_{n}\right) x_{0} \mid \xi_{n} \notin F_{0}, n=1,2, \cdots\right]
$$

such that $X_{0}$ is the linear closed extension of $S$. The $T(\xi)$ transform of any finite linear combination of elements of $S$, namely,

(3) $T(\xi)\left[a_{0} x_{0}+\sum a_{n} T\left(\xi_{n}\right) x_{0}\right]=a_{0} T(\xi) x_{0}+\sum a_{n} T\left(\xi+\xi_{n}\right) x_{0}$,

Presented to the Society, April 29, 1950; received by the editors February 13, 1950.

${ }^{1}$ Numbers in brackets refer to the references cited at the end of the paper. 
will again belong to $X_{0}$ if $\xi \notin F_{0}$ and $\xi+\xi_{n} \notin F_{0}$ for any $n$. Let $F_{n}=$ $F_{0}-\xi_{n}$ and

$$
E_{0}=\bigcup_{n=0}^{\infty} F_{n} ;
$$

then $E_{0}$ will be of measure zero. Clearly any element of the type (3) will belong to $X_{0}$ if only $\xi \notin E_{0}$. By hypothesis, $T(\xi)$ is a continuous transformation so that any limit of elements of the type $a_{0} x_{0}$ $+\sum a_{n} T\left(\xi_{n}\right) x_{0}$ will be transformed by $T(\xi)\left(\xi \notin E_{0}\right)$ into an element of $X_{0}$. Finally since the finite linear combinations of $S$ are dense in $X_{0}$, the lemma follows.

THEOREM. Let $T(\xi)$ be a strongly measurable one-parameter semigroup of operators in $\xi(X)$, then $\|T(\xi)\|$ is bounded in each interval $[\delta, 1 / \delta]$.

Suppose on the contrary that $\|T(\xi)\|$ is not bounded for some $[\delta, 1 / \delta]$. Then there exist $\xi_{n} \in[\delta, 1 / \delta]$ such that

$$
\left\|T\left(\xi_{n}\right)\right\|>n, \quad n=1,2,3, \cdots .
$$

Hence there exist $x_{n} \in X$ of norm one such that

$$
\left\|T\left(\xi_{n}\right) x_{n}\right\|>n, \quad n=1,2,3, \cdots .
$$

For each $x_{n}$ there exists, by Lemma 1, a separable closed linear subspace $X_{n}$ containing $x_{n}$ and a set $E_{n}$ of measure zero such that $T(\xi) X_{n}$ $\subset X_{n}$ for $\xi \notin E_{n}$. We define

$$
\begin{aligned}
X_{\infty} & \equiv \text { linear closed extension }\left[X_{n} \mid n=1,2, \cdots\right], \\
E_{\infty} & \equiv \bigcup_{n=1}^{\infty} E_{n} .
\end{aligned}
$$

Then $X_{\infty}$ is again a separable closed linear subspace of $X$ and $E_{\infty}$ is of measure zero. Further for any finite linear combination of $y_{n} \in X_{n}$, $T(\xi)\left[\sum a_{n} y_{n}\right] \in X_{\infty}$ if only $\xi \notin E_{\infty}$. Since the set of such finite linear combinations is dense in $X_{\infty}$ and since $T(\xi)$ is continuous, it follows that

$$
T(\xi) X_{\infty} \subset X_{\infty}
$$

if only $\xi \notin E_{\infty}$. We next define

$$
\|T(\xi)\|^{\prime}=\operatorname{LUB}\left[\|T(\xi) x\| \mid x \in X_{\infty},\|x\|=1\right] .
$$

Since $x_{n} \in X_{\infty}$, the inequality (5) implies

$$
\left\|T\left(\xi_{n}\right)\right\|^{\prime}>n \text {. }
$$


Let $\left\{z_{n}\right\}$ be a denumerable set dense on the unit sphere in $X_{\infty}$. Then since $\left\|T(\xi) z_{n}\right\|$ is a measurable real-valued function,

$$
\|T(\xi)\|^{\prime}=\operatorname{LUB}\left[\left\|T(\xi) z_{n}\right\| \mid n=1,2,3, \cdots\right]
$$

is likewise measurable. Finally if $\xi_{2} \notin E_{\infty}$, then for $x \in X_{\infty}, T\left(\xi_{2}\right) x \in X_{\infty}$ and $\left\|T\left(\xi_{2}\right) x\right\| \leqq\left\|T\left(\xi_{2}\right)\right\| \prime\|x\|$. Hence for $\xi_{2} \notin E_{\infty}$

$$
\begin{aligned}
\left\|T\left(\xi_{1}+\xi_{2}\right)\right\|^{\prime} & =\operatorname{LUB}\left[\left\|T\left(\xi_{1}\right)\left[T\left(\xi_{2}\right) x\right]\right\| \mid x \in X_{\infty},\|x\|=1\right] \\
& \leqq \operatorname{LUB}\left[\left\|T\left(\xi_{1}\right) y\right\| \mid y \in X_{\infty},\|y\|=\left\|T\left(\xi_{2}\right)\right\|^{\prime}\right] \\
& \leqq\left\|T\left(\xi_{1}\right)\right\|^{\prime} \cdot\left\|T\left(\xi_{2}\right)\right\|^{\prime} .
\end{aligned}
$$

Let $g(\xi)=\log \|T(\xi)\|^{\prime}$. Then (a) $g(\xi)$ is measurable, (b) $g(\xi)<\infty$ for each $\xi>0$, (c) $g\left(\xi_{1}+\xi_{2}\right) \leqq g\left(\xi_{1}\right)+g\left(\xi_{2}\right)$ if only $\xi_{1}$ or $\xi_{2} \notin E_{\infty}$, and (d) $g\left(\xi_{n}\right)>\log n$ where $\xi_{n} \in[\delta, 1 / \delta]$. The theorem now follows from the following lemma.

Lemma 2. If $g(\xi)$ is a measurable real-valued function on $(0, \infty)$ such that $g(\xi)<\infty$ for each $\xi>0$ and $g\left(\xi_{1}+\xi_{2}\right) \leqq g\left(\xi_{1}\right)+g\left(\xi_{2}\right)$ for $\xi_{1}$ or $\xi_{2} \notin E$ of measure zero, then $g(\xi)$ is bounded above in each interval $[\delta, 1 / \delta]$.

We omit the proof since essentially the same argument as that given by Hille [2, Theorem 6.4.1] will suffice.

EXAMPLE. In order to show that strong measurability cannot be replaced by weak measurability in the hypothesis to the above theorem, we have constructed the following example. Let the $B$-space $X$ be the nonseparable Hilbert space of complex-valued functions $x(t)$ on $(0, \infty)$ such that $\sum|x(t)|^{2}<\infty$ with norm

$$
\|x\|=\left[\sum|x(t)|^{2}\right]^{1 / 2} \text {. }
$$

Choose for $F(\xi)$ a nonmeasurable real-valued multiplicative function on $(0, \infty)$. Then

$$
F\left(\xi_{1}+\xi_{2}\right)=F\left(\xi_{1}\right) F\left(\xi_{2}\right)
$$

and $F(\xi)$ is unbounded in every finite subinterval of $(0, \infty)$ (see [3]). We then define the one-parameter semi-group of linear bounded transformations

$$
T(\xi) x(t)=F(\xi) x(t+\xi) .
$$

Clearly $\|T(\xi)\|=|F(\xi)|$ is unbounded in every subinterval of $(0, \infty)$. On the other hand, $T(\xi)$ is weakly measurable. For let $y \in \bar{X}=X$, then

$$
y[T(\xi) x]=\sum y(t) x(t+\xi) F(\xi)
$$


differs from zero only on a denumerable set of $\xi$ 's.

\section{REFERENCES}

1. Nelson Dunford, On one-parameter groups of linear transformations, Ann. of Math. vol. 39 (1938) pp. 569-573.

2. Einar Hille, Functional analysis and semi-groups, Amer. Math. Soc. Colloquium Publications, vol. 31, New York, 1948.

3. Emile Picard, Leģons sur quelques equations fonctionnelles, Paris, 1928, p. 3.

The University of Southern California

\section{MEAN VALUES AND FRULLANI INTEGRALS}

\section{RALPH PALMER AGNEW}

1. Introduction. Let $I(a, b)$ denote the Frullani integral defined by

$$
\begin{aligned}
I(a, b) & =\int_{0}^{\infty} \frac{f(a t)-f(b t)}{t} d t \\
& =\lim _{\epsilon \rightarrow 0, h \rightarrow \infty} \int_{\epsilon}^{h} \frac{f(a t)-f(b t)}{t} d t \\
& =\lim _{\epsilon \rightarrow 0, h \rightarrow \infty}\left[\int_{a \epsilon}^{a_{h}} \frac{f(t)}{t} d t-\int_{b \epsilon}^{b h} \frac{f(t)}{t} d t\right] \\
& =\lim _{h \rightarrow \infty} \int_{b h}^{a_{h}} \frac{f(t)}{t} d t-\lim _{\epsilon \rightarrow 0} \int_{b \epsilon}^{a_{\epsilon}} \frac{f(t)}{t} d t
\end{aligned}
$$

when the limits exist; it is assumed that $a, b>0$ and $f(t)$ is Lebesgue integrable over each interval $0<m \leqq t \leqq M<\infty$. If in the last two integrals we put $t=e^{u}$ and $t=e^{-u}$ respectively, and set

$$
\lambda=\log (a / b)
$$

to simplify formulas, we find that

$$
I(a, b)=\lim _{A \rightarrow \infty} \int_{A}^{\lambda+A} f\left(e^{u}\right) d u+\lim _{B \rightarrow \infty} \int_{B}^{-\lambda+B} f\left(e^{-u}\right) d u
$$

when the limits exist. From (3) we obtain immediately the Frullani formula

$$
I(a, b)=\lambda\left[\lim _{x \rightarrow \infty} f(x)-\lim _{x \rightarrow 0} f(x)\right]
$$

whenever these limits exist.

Presented to the Society, April 29, 1950; received by the editors April 10, 1950. 